CAFPE-16/03, UG-FT-146/03

DFPD 03/TH/07

IPPP $/ 03 / 04$, DCPT $/ 03 / 08$

hep-th/0302023

\title{
Bulk fields with general brane kinetic terms
}

\author{
F. del Aguila ${ }^{a}$, M. Pérez-Victoria ${ }^{a, b}$ and J. Santiago ${ }^{c}$ \\ ${ }^{a}$ Departamento de Física Teórica y del Cosmos and Centro Andaluz de \\ Física de Partículas Elementales (CAFPE), Universidad de Granada, \\ E-18071 Granada, Spain \\ ${ }^{b}$ Dipartimento di Fisica "G. Galilei", Università di Padova and INFN, \\ Sezione di Padova, Via Marzolo 8, I-35131 Padua, Italy \\ ${ }^{c}$ Institute for Particle Physics Phenomenology, University of Durham, \\ South Road, Durham DH1 3LE, UK
}

\begin{abstract}
We analyse the effect of general brane kinetic terms for bulk scalars, fermions and gauge bosons in theories with extra dimensions, with and without supersymmetry. We find in particular a singular behaviour when these terms contain derivatives orthogonal to the brane. This is brought about by $\delta(0)$ divergences arising at second and higher order in perturbation theory. We argue that this behaviour can be smoothed down by classical renormalization.
\end{abstract}




\section{Introduction}

Field theoretical models with extra dimensions typically include one or more lower dimensional defects on which some fields can be localized. These defects ("branes" from now on) can have different microscopic realizations: Dbranes, domain walls, cosmic strings, orbifold fixed points, ... In general, the fields propagating in all the dimensions (bulk fields) couple to fields propagating on the branes [1, 2. Moreover, it is possible that the action includes terms localized on the branes involving only bulk fields ("brane terms", for short). They may have important consequences. A crucial observation is that brane terms are induced by radiative corrections. This is allowed as Poincaré invariance is broken by the branes. Quantum divergences localized on the branes can appear in the correlators of bulk fields when the bulk fields are coupled to fields living on them [3]. Furthermore, in orbifolds this can occur even in the absence of brane couplings at tree level [4. In any case, it is clear that the presence of divergent radiative corrections localized on the branes requires the introduction of divergent brane counterterms. Therefore, the corresponding brane couplings run with the renormalization scale, and cannot be set to zero at all scales. Furthermore, this indicates that they should be considered free parameters of the theory and be included at tree level (see [5] for a detailed argument). Of course, since interacting theories in higher dimensions are nonrenormalizable, these considerations are only meaningful in the framework of effective field theory, and it is important to take into account possible suppressions of the brane couplings by inverse powers of the cutoff scale $\Lambda$.

Brane terms are always suppressed by $1 / \Lambda^{n}, n \geq 1$, with respect to the analogous bulk terms. Therefore one would expect that their effect on observables should be a small perturbation of the corresponding results without brane terms. In this article we show that, contrary to this expectation, certain brane operators modify severely some observables-like Kaluza-Klein (KK) masses - even when their coefficients are very small. This breakdown of perturbation theory has to do with the interplay between the thin brane limit and the limit of large $\Lambda$, in which the coefficients of the brane operators 
approach zero. Unless otherwise stated, we will always consider infinitely thin, delta-function branes, although we will often make use of thick branes as intermediate regulators. On the other hand, the underlying fundamental theory is usually assumed to be smooth in its parameters, so that all its low energy predictions should be perturbatively calculable using some effective theory. From this point of view, the relevant question is how to incorporate in a low energy effective description a mechanism to make the expansion in $\Lambda$ well defined. Here we sketch a possible answer in the spirit of perturbative renormalization theory.

We shall concentrate on brane kinetic terms. Kinetic terms localized on a brane were first invoked by Dvali, Gabadadze and Porrati [3 to recover 4D gravitational behaviour at short distances in infinite extra dimensions. The same mechanism was subsequently applied in [6] to gauge theories. In [7], the one loop radiative corrections to brane terms and their first order impact on KK spectra were calculated in different theories. In [8], a detailed computation of infinite and finite radiative corrections to bulk and brane kinetic terms of gauge fields (with and without Hosotani mechanism) was performed. The phenomenology of gauge fields in compact dimensions with (tree-level) brane kinetic terms has been discussed recently in [5] (flat space) and [9, 10] (warped space). A particular fermionic case has been addressed in [11. Finally, brane kinetic terms in supersymmetric models have been studied in [12, 13, 14, 15. In all these works, brane kinetic terms are found to have a significant impact on model building and phenomenology. And, as observed above, they must be generically included for consistency.

Here we analyse at the classical level the effect of all the possible $\mathcal{O}(1 / \Lambda)$ brane kinetic terms for scalar, fermion and gauge fields. In particular we examine brane terms containing derivatives with respect to the coordinates orthogonal to the brane. These terms have not been studied in detail before ${ }^{1}$. We find that they give rise to solutions to the equations of motion which exhibit a rather singular behaviour: they decouple from the brane and become insensitive to the magnitude of all brane couplings; furthermore, physical

\footnotetext{
${ }^{1}$ Except in [16, where the singular terms were "massaged" via a classical renormalization. We compare this approach with ours in Section [5]
} 
observables for arbitrarily small coefficients of these terms do not approach the ones for vanishing coefficients. This is related to the appearance of $\delta(0)$ singularities at second and higher orders in the $1 / \Lambda$ expansion. These singularities are a common feature of field theoretical models with infinitely thin branes [17, 2]. We also study brane kinetic terms in supersymmetric theories and find that supersymmetry requires the presence of higher order kinetic terms in the on-shell action. These terms improve but do not cure the singular behaviour of the classical fields. More generally, any effective theory will contain higher order terms which may significantly change the solutions derived from the first order Lagrangian. One can then hope that these terms be such that the exact solutions are smooth in all the parameters of the theory. We will discuss this possibility below. Although most of our considerations apply to general field theories in extra dimensions, for definiteness we study here theories defined on a flat 5D space with the 5th dimension compatified on an $S^{1} / Z_{2}$ orbifold. Moreover, in order to keep the discussion and calculations as simple as possible, we neglect masses and consider kinetic terms localized on only one of the fixed points (even if kinetic terms on both branes are natural and lead to interesting phenomenology [ [5] $)$.

The structure of the paper is as follows. In Section 2 we perform the exact KK reduction of the free action for particles of spin $\leq 1$ in the presence of general brane kinetic terms. In Section 3 we study the supersymmetric case. In Section 4 we examine in detail an example elucidating the role of thin brane singularities. In Section 5 we argue that classical renormalization makes the theory smooth and perturbation theory well defined. This is also interpreted as the restriction to a subclass of effective theories with particular reductions of couplings. We summarize and discuss our results in Section 6. Finally, we collect in Appendix A the general first order results, and give an example of renormalization in Appendix B.

\section{Exact KK reduction}

In this section we study the exact (i.e., nonperturbative) KK reduction of the most general free action with leading order brane terms for massless 
scalars, gauge bosons and fermions propagating in $M_{4} \times S^{1} / Z_{2}$. We consider fields which are functions of the coordinates $x^{\mu}, \mu=0,1,2,3$ of $M_{4}$ and $y \in(-\pi R, \pi R]$, of $S^{1}$, and which are eigenstates of $Z_{2}$, i.e., even or odd functions of $y$. For simplicity, we only include brane terms at the fixed point $y=0$.

Some of the operators we consider are products of two odd factors times a delta function. These terms are sometimes argued to vanish on parity grounds. However, these arguments are no longer valid when the (derivatives of) wave functions appearing in these terms develop discontinuities across the branes [18. Deriving and solving the equations of motion when the action contains this sort of terms is tricky [19], and the same applies to brane terms containing derivatives with respect to $y$.

In order to treat all these cases rigorously we regularize the branes using several (smooth) representations of the delta function, of width $\sim \epsilon$. We perform all the calculations with these resolved branes, and only take the thin brane limit $\epsilon \rightarrow 0$ at the very end. Furthermore, we employ different representations in order to check that our results are regularization independent. In some cases we have resorted to numerical methods to solve the relevant differential equations. Still, it is possible to see that the numerical solutions converge rapidly in the thin brane limit to certain functions, which we take as our analytical solutions. The reader can find an explicit analytical calculation in the simple case discussed in Section 4.

\section{$2.1 \quad$ Scalars}

The most general action for a massless complex five-dimensional scalar with leading order brane kinetic terms is ${ }^{2}$

$$
\begin{array}{r}
S=\int \mathrm{d}^{4} x \\
\int_{-\pi R}^{\pi R} \mathrm{~d} y\left\{\left(1+a \delta_{0}\right) \partial_{\mu} \phi^{\dagger} \partial^{\mu} \phi-\partial_{y} \phi^{\dagger} \partial_{y} \phi\right. \\
\left.+\delta_{0}\left[\frac{b}{2}\left(\phi^{\dagger} \partial_{y}^{2} \phi+\partial_{y}^{2} \phi^{\dagger} \phi\right)-c \partial_{y} \phi^{\dagger} \partial_{y} \phi\right]\right\}
\end{array}
$$

\footnotetext{
${ }^{2}$ More generally we could consider a term $\delta_{0}\left(\frac{b}{2} \phi^{\dagger} \partial_{y}^{2} \phi+\frac{b^{*}}{2} \partial_{y}^{2} \phi^{\dagger} \phi\right)$, but we take $b$ real for simplicity.
} 
where $\delta_{0} \equiv \delta(y)$. The dimensionful parameters $a, b$ and $c$ are expected to be of order $1 / \Lambda$. In order to perform the KK reduction we expand

$$
\phi(x, y)=\sum_{n=0}^{\infty} \frac{f_{n}(y)}{\sqrt{2 \pi R}} \phi^{(n)}(x),
$$

where $\left\{f_{n}\right\}$ is some basis of functions on $(-\pi R, \pi R]$ with the same parity character as $\phi$. Introducing (2.2) in the action we find

$$
\begin{aligned}
S=\int \mathrm{d}^{4} x & \sum_{m n}\left\{\int_{-\pi R}^{\pi R} \mathrm{~d} y\left(1+a \delta_{0}\right) \frac{f_{m} f_{n}}{2 \pi R} \partial_{\mu} \phi^{(m) \dagger} \partial^{\mu} \phi^{(n)}\right. \\
& \left.+\int \mathrm{d} y \frac{f_{m} \mathcal{O} f_{n}}{2 \pi R} \phi^{(m) \dagger} \phi^{(n)}\right\}
\end{aligned}
$$

where we have defined

$$
\mathcal{O} \equiv\left[1+(b+c) \delta_{0}\right] \partial_{y}^{2}+(b+c) \delta_{0}^{\prime} \partial_{y}+\frac{b}{2} \delta_{0}^{\prime \prime} .
$$

In order to diagonalize the quadratic terms, we take $\left\{f_{n}\right\}$ as the set of eigenfunctions of the following generalised eigenvalue problem:

$$
\mathcal{O} f_{n}=-m_{n}^{2}\left(1+a \delta_{0}\right) f_{n}
$$

The fact that the operator $\mathcal{O}$ is hermitian and that the eigenvalues of (2.5) are nondegenerate, ensures the orthogonality of eigenfunctions with respect to the scalar product

$$
<f, g>=\frac{1}{2 \pi R} \int_{-\pi R}^{\pi R} \mathrm{~d} y\left(1+a \delta_{0}\right) f(y) g(y) .
$$

Then, if we also impose, when possible, the normalization

$$
<f_{n}, f_{n}>=1
$$

the free action reduces to

$$
S=\int \mathrm{d}^{4} x \sum_{n}\left\{\partial_{\mu} \phi^{(n) \dagger} \partial^{\mu} \phi^{(n)}-m_{n}^{2} \phi^{(n) \dagger} \phi^{(n)}\right\} .
$$


The generalised eigenvalue equation (2.5) is to be solved with the appropriate orbifold constraints.

When $b<0$ or $c<0$, it turns out that, for finite (small enough) $\epsilon$, the eigenfunctions diverge at some point near $y=0$. For this reason, we must require $b \geq 0, c \geq 0$ to have a well defined theory.

Let us now describe the exact solutions in the thin brane limit. For odd scalars, the wave functions and KK masses are insensitive to the brane terms. The wave functions are

$$
f_{n}^{\text {odd }}(y)=\sqrt{2} \sin \left(m_{n} y\right),
$$

and the masses, $m_{n}=n / R$, with $n$ a positive integer ${ }^{3}$.

For even scalars, the wave functions and masses do not depend on the (positive) coefficient $c$ when $\epsilon \rightarrow 0$. We distinguish two cases:

- $b=0$. In this case, the solution coincides with the one described in [5]. There is a constant massless mode, which depends on the brane terms only through its normalization:

$$
f_{0}=\frac{1}{\sqrt{1+\frac{a}{2 \pi R}}} .
$$

The massive modes are given by

$$
f_{n}(y)=A_{n}\left[\cos \left(m_{n} y\right)-\frac{a m_{n}}{2} \sin \left(m_{n}|y|\right)\right],
$$

where $A_{n}$ is a normalization constant and the masses are the solutions of the equation

$$
\tan \left(\pi R m_{n}\right)+\frac{a}{2} m_{n}=0 .
$$

We observe that if $-2 \pi R \leq a<0$, there is a tachyonic mode, with squared mass approaching $-4 / a^{2}$ for small $|a| /(2 \pi R)$. On the other hand, the norm squared defined by the scalar product (2.6), $\|f\|^{2}=$ $<f, f>$, is indefinite. This can be problematic, since zero norm modes

\footnotetext{
${ }^{3}$ The derivatives of the wave functions, however, have discontinuous values at $y=0$ in the limit $\epsilon \rightarrow 0$, whenever $c \neq 0: f_{n}^{\prime}(0)=0 \neq f_{n}^{\prime}\left(0^{+}\right)$. This behaviour at the brane is the same as the one for the even component of a fermion, to be described below.
} 
have vanishing kinetic term in the reduced $4 \mathrm{D}$ action (they cannot be normalized as in (2.7)), while modes with negative norm squared have the wrong sign in the reduced kinetic term (they are ghosts). We find that if $a=-2 \pi R$ the zero mode has zero norm, and if $a<-2 \pi R$ it has negative norm squared (the massive modes have always positive norm). Therefore, we see that for negative values of $a$ the theory is either pathological or unstable.

- $b>0$. In this case the solution is independent of all brane terms, including $b$ itself (as long as it is strictly positive). We find

$$
f_{n}(y)=A_{n} \sin \left(m_{n}|y|\right)
$$

with $m_{n}=\frac{n+1 / 2}{R}$ and $n$ integer. In particular, there is no zero mode.

We see that the KK spectrum and wave functions of even scalars change dramatically when an arbitrarily small $b$ is turned on. Note also that the wave functions vanish at $y=0$ when $b>0$.

\subsection{Gauge Bosons}

The Lagrangian for gauge bosons admits brane kinetic terms analogous to the ones for scalars, but with the restrictions of gauge invariance. Since we are only discussing the quadratic action, we can consider an abelian theory without loss of generality. For a gauge boson $A_{M}$ with general $\mathcal{O}(1 / \Lambda)$ kinetic terms on the first brane,

$$
S=\int \mathrm{d}^{4} x \int \mathrm{d} y\left\{-\frac{1}{4}\left(1+a \delta_{0}\right) F_{\mu \nu} F^{\mu \nu}-\frac{1}{2}\left(1+c \delta_{0}\right) F_{5 \nu} F^{5 \nu}\right\} .
$$

The bulk and brane parts respect $5 \mathrm{D}$ and $4 \mathrm{D}$ Lorentz symmetry, respectively. The orbifold projection forces $A_{\mu}$ and $A_{5}$ to have opposite $Z_{2}$ parity. The whole Lagrangian is symmetric under the gauge transformations $A_{M} \rightarrow A_{M}+$ $\partial_{M} \theta$, where the gauge parameter $\theta$ has the same parity as $A_{\mu}$. Using a gauge $A_{5}=0$ or $\partial_{M} A^{M}=0$ it is easy to see that the equations of motion are the same as the ones for scalars without $b$ terms. At the level of the action, these 
gauges can be imposed adding the corresponding gauge fixing terms. To perform the KK decomposition, however, we choose to work with the gauge invariant action. We expand

$$
\begin{aligned}
& A_{\mu}(x, y)=\sum_{n=0}^{\infty} \frac{f_{n}(y)}{\sqrt{2 \pi R}} A_{\mu}^{(n)}(x), \\
& A_{5}(x, y)=\sum_{n=0}^{\infty} \frac{\partial_{y} f_{n}(y)}{m_{n} \sqrt{2 \pi R}} A_{5}^{(n)}(x),
\end{aligned}
$$

with $A_{5}^{(0)}=0$ if $m_{0}=0$, and require

$$
\mathcal{O} f_{n}=-m_{n}^{2}\left(1+a \delta_{0}\right) f_{n}
$$

with

$$
\mathcal{O} \equiv\left(1+c \delta_{0}\right) \partial_{y}^{2}+c \delta_{0}^{\prime} \partial_{y} .
$$

Thanks to the form of the expansion of $A_{5}$ and the hermiticity of the differential operator $\mathcal{O}$, all the terms get diagonalized. To have canonical $4 \mathrm{D}$ kinetic terms we also impose the normalization condition (2.7). The eigenfunctions and eigenmasses can be read directly from the results for (even or odd) scalars with $b=0$. Again, there is no well defined solution for $c<0$, so we require $c \geq 0$. And for $a<0$ we have either tachyons or ghosts.

At the end we are left with the following $4 \mathrm{D}$ Lagrangian:

$\mathcal{L}=\sum_{n=0}^{\infty}\left\{-\frac{1}{4} F_{\mu \nu}^{(n)} F^{\mu \nu(n)}+\frac{1}{2} \partial_{\mu} A_{5}^{(n)} \partial^{\mu} A_{5}^{(n)}+\frac{1}{2} m_{n}^{2} A_{\mu}^{(n)} A^{(n) \mu}+m_{n} A_{5}^{(n)} \partial_{\mu} A^{(n) \mu}\right\}$.

This Lagrangian is invariant under an infinite set of $4 \mathrm{D}$ local transformations:

$$
\begin{aligned}
& A_{\mu}^{(n)} \rightarrow A_{\mu}^{(n)}+\partial_{\mu} \theta^{(n)}, \\
& A_{5}^{(n)} \rightarrow A_{5}^{(n)}+m_{n} \theta^{(n)},
\end{aligned}
$$

which is nothing but the KK decomposition of 5D gauge transformations:

$$
\theta(x, y)=\sum_{n=0}^{\infty} \frac{f_{n}(y)}{\sqrt{2 \pi R}} \theta^{(n)}(x) .
$$


The scalars $A_{5}^{(n)}$ can be interpreted as Goldstone bosons of the spontaneously broken gauge transformations $\theta^{(n)}$ (those with $f_{n}(y) \neq$ constant). They can be decoupled from the $4 \mathrm{D}$ vector bosons $A_{\mu}^{(n)}$ using an $R_{\xi}$ gauge. Indeed, adding to (2.19) the gauge fixing terms

$$
-\frac{1}{2 \xi} \sum_{n=0}^{\infty}\left(\partial_{\mu} A^{(n) \mu}+\xi m_{n} A_{5}^{(n)}\right)^{2}
$$

we find

$$
\begin{aligned}
\mathcal{L}^{\prime}=\sum_{n=0}^{\infty}\{ & -\frac{1}{4} F_{\mu \nu}^{(n)} F^{\mu \nu(n)}-\frac{1}{2 \xi}\left(\partial_{\mu} A^{(n) \mu}\right)^{2}+\frac{1}{2} m_{n}^{2} A_{\mu}^{(n)} A^{(n) \mu}+\frac{1}{2} \partial_{\mu} A_{5}^{(n)} \partial^{\mu} A_{5}^{(n)} \\
& \left.-\xi m_{n}^{2}\left(A_{5}^{(n)}\right)^{2}\right\} .
\end{aligned}
$$

In the Feynman-'t Hooft gauge, $\xi=1$, the scalar fields $A_{5}^{(n)}$ have mass $m_{n}$, whereas in the unitary gauge, $\xi \rightarrow \infty$, these fields get infinite masses and decouple from the spectrum.

\section{$2.3 \quad$ Fermions}

Let us consider now the most general kinetic action for a five-dimensional bulk fermion $\psi=\psi_{L}+\psi_{R}$, with $\gamma^{5} \psi_{L, R}=\mp \psi_{L, R}$, and leading order brane contributions,

$$
\begin{gathered}
S=\int \mathrm{d}^{4} x \int_{-\pi R}^{\pi R} \mathrm{~d} y\left\{\left(1+a^{L} \delta_{0}\right) \bar{\psi}_{L} \mathrm{i} \not \partial \psi_{L}+\left(1+a^{R} \delta_{0}\right) \bar{\psi}_{R} \mathrm{i} \not \partial \psi_{R}\right. \\
-\left(1+\frac{b}{2} \delta_{0}\right) \bar{\psi}_{L} \partial_{y} \psi_{R}-\frac{b}{2} \delta_{0}\left(\partial_{y} \bar{\psi}_{R}\right) \psi_{L} \\
\left.+\left(1+\frac{c}{2} \delta_{0}\right) \bar{\psi}_{R} \partial_{y} \psi_{L}+\frac{c}{2} \delta_{0}\left(\partial_{y} \bar{\psi}_{L}\right) \psi_{R}\right\} .
\end{gathered}
$$

We have considered again real $b$ and $c$. The two chiralities have opposite parity. We take $\psi_{L}$ even for definiteness. The KK expansion of the fermions reads

$$
\psi_{L, R}(x, y)=\sum_{n} \frac{f_{n}^{L, R}(y)}{\sqrt{2 \pi R}} \psi_{L, R}^{(n)}(x) .
$$


In order to diagonalize the $4 \mathrm{D}$ kinetic and mass terms, we take the wave functions $f_{n}^{L, R}$ to be the eigenfunctions of the following generalised eigenvalue problem:

$$
\begin{aligned}
& \mathcal{O}_{1} f_{n}^{R}=m_{n}\left(1+a^{L} \delta_{0}\right) f_{n}^{L}, \\
& \mathcal{O}_{2} f_{n}^{L}=-m_{n}\left(1+a^{R} \delta_{0}\right) f_{n}^{R},
\end{aligned}
$$

where

$$
\begin{aligned}
& \mathcal{O}_{1}=\left[1+\frac{1}{2}(b+c) \delta_{0}\right] \partial_{y}+\frac{c}{2} \delta_{0}^{\prime}, \\
& \mathcal{O}_{2}=\left[1+\frac{1}{2}(b+c) \delta_{0}\right] \partial_{y}+\frac{b}{2} \delta_{0}^{\prime} .
\end{aligned}
$$

Because $\mathcal{O}_{1}^{\dagger}=-\mathcal{O}_{2}$, the functions $f_{n}^{L, R}$ are orthogonal with respect to the appropriate scalar products,

$$
<f, g>_{L, R}=\frac{1}{2 \pi R} \int_{-\pi R}^{\pi R} \mathrm{~d} y\left(1+a^{L, R} \delta_{0}\right) f(y) g(y) .
$$

We also impose the normalization conditions

$$
<f_{n}^{L}, f_{n}^{L}>_{L}=<f_{n}^{R}, f_{n}^{R}>_{R}=1
$$

to render the $4 \mathrm{D}$ kinetic terms canonically normalized.

As in previous cases, we must require $b, c \geq 0$ to have well defined solutions. The solutions then depend on whether $b$ vanishes or not:

- $b=0$. There is a chiral zero mode with $f^{R}=0$ and a flat left-handed wave function,

$$
f_{0}^{L}=\frac{1}{\sqrt{1+\frac{a^{L}}{2 \pi R}}} .
$$

When $c=0$, the wave functions for massive modes are ${ }^{4}$

$$
\begin{aligned}
& f_{n}^{L}=B_{n}\left[\cos \left(m_{n} y\right)+\tan \left(m_{n} \pi R\right) \sin \left(m_{n}|y|\right)\right], \\
& f_{n}^{R}=C_{n}\left[\sin \left(m_{n} y\right)-\sigma(y) \tan \left(m_{n} \pi R\right) \cos \left(m_{n} y\right)\right],
\end{aligned}
$$

\footnotetext{
${ }^{4}$ Strictly, there is no uniform convergence as $\epsilon \rightarrow 0$ due to the behaviour around $y=0$. We write the limiting functions for $|y|>\eta$, with $\eta$ an arbitrarily small positive number, keeping in mind that the interactions of the wave functions with the brane are to be calculated before taking the limit $\epsilon \rightarrow 0$.
} 
with $\sigma$ the sign function and masses given by

$$
\tan \left(\pi R m_{n}\right)+\sqrt{\frac{a^{L}}{a^{R}}} \tan \left(\sqrt{a^{L} a^{R}} \frac{m_{n}}{2}\right)=0 .
$$

These solutions reduce to the ones for even scalar fields when $a^{R}=0$. Note also that $a^{R}$ is irrelevant when $a^{L}=0$. The reason is that only when $a^{L}$ forces a discontinuity at the brane is the "odd-odd" $a^{R}$ term nonvanishing for $\epsilon \rightarrow 0$. When $c>0$, the solutions reduce again to the ones with $a^{R}=0$, i.e., the only effect of a positive $c$ is to cancel the effect of $a^{R}$.

- $b>0$. In this case, the wave functions (for $|y|>\eta>0$, see previous footnote) and masses reduce to the ones without brane terms: There is a chiral zero mode

$$
f_{0}^{L}=1
$$

and a tower of massive modes

$$
\begin{aligned}
& f_{n}^{L}=B_{n} \cos \left(m_{n} y\right), \\
& f_{n}^{R}=B_{n} \sin \left(m_{n} y\right),
\end{aligned}
$$

with $m_{n}=n / R$. In all cases, $f_{n}(0)=0$, so that the functions are not piecewise continuous. More precisely,

$$
\lim _{\epsilon \rightarrow 0} \int \mathrm{d} y \delta_{\epsilon}(y) f_{\epsilon n}^{L}(y)=0
$$

Again, we see that the solutions change abruptly when $b$ or $c$ are turned on. Moreover, when $b>0$ the wave functions vanish at $y=0$, so the fermions do not couple to brane fields.

\section{Brane kinetic terms in supersymmetric the- ories}

In this section we study the impact of brane kinetic terms in supersymmetric theories. Besides the intrinsic interest of these theories, one might hope that 
supersymmetry improves the singular behaviour we have observed in the previous section. One more reason to look at supersymmetric models is the following: we have seen that bosons and fermions with brane kinetic terms have different KK wave functions and masses; an intriguing question is then how supersymmetry makes the spectra of bosons and fermions in the same supermultiplet identical.

We consider the supersymmetric quadratic actions of massless 5D hyper and vector multiplets including all possible $\mathcal{O}(1 / \Lambda)$ brane kinetic terms.

\subsection{Hypermultiplet}

Off shell, the 5D hypermultiplet contains two complex scalars, $\phi_{1,2}$, a Dirac fermion $\psi$ (with two Weyl components, $\psi_{1,2}$ ), and two complex auxiliary fields, $F_{1,2}$. We want to construct an action for these fields, invariant under $5 \mathrm{D} \mathrm{N}=1$ supersymmetry in the bulk and under 4D $\mathrm{N}=1$ supersymmetry on the brane. It is easy to write such an action in terms of two $4 \mathrm{D} N=1$ superfields [20, 21]. The Lagrangian is

$$
\mathcal{L}=\mathcal{L}_{\text {bulk }}+\mathcal{L}_{\text {brane }}
$$

with

$$
\mathcal{L}_{\text {bulk }}=\int d^{4} \theta\left(\Phi_{1}^{\dagger} \Phi_{1}+\Phi_{2}^{\dagger} \Phi_{2}\right)+\left[\int d^{2} \theta \Phi_{1} \partial_{y} \Phi_{2}+\text { h.c. }\right]
$$

and

$$
\begin{aligned}
\mathcal{L}_{\text {brane }}=\delta_{0} & \left\{\int d^{4} \theta\left(a_{1} \Phi_{1}^{\dagger} \Phi_{1}+a_{2} \Phi_{2}^{\dagger} \Phi_{2}\right)\right. \\
+ & \left.\left.+\int d^{2} \theta\left(b \Phi_{1} \partial_{y} \Phi_{2}-c \Phi_{2} \partial_{y} \Phi_{1}\right)+\text { h.c. }\right]\right\},
\end{aligned}
$$

where, in the $y$ basis, $\Phi_{i}=\phi_{i}+\sqrt{2} \theta \psi_{i}+\theta^{2} F_{i}$. $\Phi_{1}$ and $\Phi_{2}$ (and their corresponding components) have opposite $Z_{2}$ parities. In this case we have allowed for complex $b$ and $c$ to keep track of the analytic properties of the supersymmetric theory. In components, the bulk and brane Lagrangians read 
(summation over $i$ is undertood)

$$
\begin{aligned}
\mathcal{L}_{\text {bulk }}= & -\phi_{i}^{\dagger} \square \phi_{i}+i \partial_{\mu} \bar{\psi}_{i} \bar{\sigma}^{\mu} \psi_{i}+F_{i}^{\dagger} F_{i} \\
& +\left[\phi_{1} \partial_{y} F_{2}+F_{1} \partial_{y} \phi_{2}-\psi_{1} \partial_{y} \psi_{2}+\text { h.c. }\right], \\
\mathcal{L}_{\text {brane }}= & \delta_{0}\left\{a_{i}\left[-\phi_{i}^{\dagger} \square \phi_{i}+i \partial_{\mu} \bar{\psi}_{i} \bar{\sigma}^{\mu} \psi_{i}+F_{i}^{\dagger} F_{i}\right]\right. \\
& +\left(b\left[\phi_{1} \partial_{y} F_{2}+F_{1} \partial_{y} \phi_{2}-\psi_{1} \partial_{y} \psi_{2}\right]\right. \\
& \left.\left.-c\left[\phi_{2} \partial_{y} F_{1}+F_{2} \partial_{y} \phi_{1}-\psi_{2} \partial_{y} \psi_{1}\right]+\text { h.c. }\right)\right\} .
\end{aligned}
$$

The equations of motion for the auxiliary fields are

$$
\begin{aligned}
& {\left[1+a_{1} \delta_{0}\right] F_{1}+\left[1+b^{*} \delta_{0}\right] \partial_{y} \phi_{2}^{\dagger}+c^{*} \partial_{y}\left[\delta_{0} \phi_{2}^{\dagger}\right]=0} \\
& {\left[1+a_{2} \delta_{0}\right] F_{2}-\left[1+c^{*} \delta_{0}\right] \partial_{y} \phi_{1}^{\dagger}-b^{*} \partial_{y}\left[\delta_{0} \phi_{1}^{\dagger}\right]=0 .}
\end{aligned}
$$

We can directly solve them, keeping in mind that the delta functions are regularized. Inserting the solutions in the action we find

$$
\begin{aligned}
\mathcal{L}=\{ & -\left(1+a_{1} \delta_{0}\right) \phi_{1}^{\dagger} \square \phi_{1}-\frac{\left|1+c \delta_{0}\right|^{2}}{1+a_{2} \delta_{0}} \partial_{y} \phi_{1}^{\dagger} \partial_{y} \phi_{1} \\
& -\left[\frac{b\left(1+c^{*} \delta_{0}\right)}{1+a_{2} \delta_{0}} \partial_{y} \phi_{1}^{\dagger} \partial_{y}\left(\delta_{0} \phi_{1}\right)+\text { h.c. }\right] \\
& \left.-\frac{|b|^{2}}{1+a_{2} \delta_{0}} \partial_{y}\left(\delta_{0} \phi_{1}^{\dagger}\right) \partial_{y}\left(\delta_{0} \phi_{1}\right)+(1 \leftrightarrow 2, b \leftrightarrow c)\right\} \\
& +\bar{\psi}_{L} i \not \partial \psi_{L}+\bar{\psi}_{R} i \not \partial \psi_{R}-\bar{\psi}_{L} \partial_{y} \psi_{R}+\bar{\psi}_{R} \partial_{y} \psi_{L} \\
& +\delta_{0}\left[a_{1} \bar{\psi}_{L} i \not \partial \psi_{L}+a_{2} \bar{\psi}_{R} i \not \partial \psi_{R}+\left(-b \partial_{y} \bar{\psi}_{R} \psi_{L}+c \bar{\psi}_{R} \partial_{y} \psi_{L}+\text { h.c. }\right)\right] .
\end{aligned}
$$

We have defined the chiral 4-component spinors $\psi_{L}^{T}=\left(\psi_{1} 0\right), \psi_{R}^{T}=\left(\begin{array}{l}0 \bar{\psi}_{2}\end{array}\right)$. The fermionic sector coincides with the one studied in Section 2 (with $b \rightarrow 2 b$, $c \rightarrow 2 c)$. That the spectra of $\phi_{1}, \phi_{2}^{\dagger}$ and $\psi_{L, R}$ are identical can be readily seen from the equations of motion for the KK wave functions. The equation for $\phi_{1}$ is

$$
\begin{aligned}
& {\left[\left|1+(b+c) \delta_{0}\right|^{2} \partial_{y}^{2}+\left[\left(1+\left(b^{*}+c^{*}\right) \delta_{0}\right)(2 b+c)+\left(1+(b+c) \delta_{0}\right) c^{*}\right.\right.} \\
& \left.\left.-\frac{a_{2}\left|1+(b+c) \delta_{0}\right|^{2}}{1+a_{2} \delta_{0}}\right] \delta_{0}^{\prime} \partial_{y}+b\left(1+\left(b^{*}+c^{*}\right) \delta_{0}\right)\left(\delta_{0}^{\prime \prime}-\frac{a_{2}\left(\delta_{0}^{\prime}\right)^{2}}{1+a_{2} \delta_{0}}\right)+b c^{*}\left(\delta_{0}^{\prime}\right)^{2}\right] f_{n}^{1} \\
& =-m_{n}^{2}\left(1+a_{1} \delta_{0}\right)\left(1+a_{2} \delta_{0}\right) f_{n}^{1}
\end{aligned}
$$


and the one for $\phi_{2}^{\dagger}$ can be obtained from this one by $1 \leftrightarrow 2$ and $b \leftrightarrow c^{*}$. For the fermions,

$$
\begin{aligned}
& \left\{\left[1+\left(b^{*}+c^{*}\right) \delta_{0}\right] \partial_{y}+c^{*} \delta_{0}^{\prime}\right\} f_{n}^{R}=m_{n}\left(1+a_{1} \delta_{0}\right) f_{n}^{L}, \\
& \left\{\left[1+(b+c) \delta_{0}\right] \partial_{y}+b \delta_{0}^{\prime}\right\} f_{n}^{L}=-m_{n}\left(1+a_{2} \delta_{0}\right) f_{n}^{R} .
\end{aligned}
$$

It is then trivial to show that iterating the fermionic equations we obtain the scalar ones and thus the spectra of fields in the same 4D N=1 supermultiplet are exactly the same, as implied by supersymmetry. We see that in order for the scalar solutions to reproduce the fermionic ones, higher order (singular) terms are required in the scalar sector. They are reminiscent of the famous $\delta(y)^{2}$ terms of [2].

This example illustrates the fact that higher order operators can modify radically the spectrum, although supersymmetry does not prevent the singular behaviour we observed in the last section for the coefficients $b$ and $c$. Nevertheless, we can consider the theory with $b=0$ and $c=0$, which behaves smoothly and looks stable under quantum corrections, as these terms are protected by nonrenormalization theorems. Note, however, that higher order D-terms, which can in principle be induced, may reintroduce the singular behaviour.

\subsection{Vector multiplet}

The 5D off-shell vector multiplet consists of a 5D vector $A_{M}$, two Weyl gauginos $\lambda_{1,2}$, a real scalar $\Sigma$, a real auxiliary field $D$ and a complex one $F_{\chi}$. Under $\mathrm{D}=4 \mathrm{~N}=1$ supersymmetry they form a vector supermultiplet, $V$, and a chiral one, $\chi[20,21]$ :

$$
\begin{aligned}
& V=-\theta \sigma^{\mu} \bar{\theta} A_{\mu}-i \bar{\theta}^{2} \theta \lambda_{1}+i \theta^{2} \bar{\theta} \bar{\lambda}_{1}+\frac{1}{2} \bar{\theta}^{2} \theta^{2} D, \\
& \chi=\frac{1}{\sqrt{2}}\left(\Sigma+i A_{5}\right)+\sqrt{2} i \theta \lambda_{2}+\theta^{2} F_{\chi},
\end{aligned}
$$

where $V$ is written in the Wess-Zumino gauge and $\chi$ in the " $y$ " basis. Since we are interested in the free action we consider an Abelian theory. The gauge 
transformations are

$$
\begin{aligned}
& V \rightarrow V+\Lambda+\Lambda^{\dagger}, \\
& \chi \rightarrow \chi+\sqrt{2} \partial_{y} \Lambda,
\end{aligned}
$$

with $\Lambda$ a chiral superfield. The gauge invariant Lagrangian including $\mathcal{O}(1 / \Lambda)$ arbitrary brane terms is $\mathcal{L}=\mathcal{L}_{\text {bulk }}+\mathcal{L}_{\text {brane }}$ with

$$
\mathcal{L}_{\text {bulk }}=-\frac{1}{4} \int \mathrm{d}^{2} \theta W^{\alpha} W_{\alpha}+\text { h.c. }+\int \mathrm{d}^{4} \theta\left(\partial_{y} V-\frac{1}{\sqrt{2}}\left(\chi+\chi^{\dagger}\right)\right)^{2},
$$

and

$$
\mathcal{L}_{\text {brane }}=\delta_{0}\left[-\frac{1}{4} a \int \mathrm{d}^{2} \theta W^{\alpha} W_{\alpha}+\text { h.c. }+c \int \mathrm{d}^{4} \theta\left(\partial_{y} V-\frac{1}{\sqrt{2}}\left(\chi+\chi^{\dagger}\right)\right)^{2}\right]
$$

The corresponding action is $5 \mathrm{D} N=1$ supersymmetric in the bulk and 4D $\mathrm{N}=1$ supersymmetric on the brane. $V$ and $\chi$ have opposite $Z_{2}$ parity. In components the Lagrangian reads

$$
\begin{aligned}
\mathcal{L}_{\text {bulk }}= & -\frac{1}{4} F^{M N} F_{M N}+\frac{1}{2} \partial^{\mu} \Sigma \partial_{\mu} \Sigma+\frac{1}{2} D^{2}-\Sigma \partial_{y} D+F_{\chi} F_{\chi}^{\dagger} \\
& -\lambda_{1} i \sigma^{\mu} \partial_{\mu} \bar{\lambda}_{1}-\lambda_{2} i \sigma^{\mu} \partial_{\mu} \bar{\lambda}_{2}+\lambda_{2} \partial_{y} \lambda_{1}+\bar{\lambda}_{2} \partial_{y} \bar{\lambda}_{1} \\
\mathcal{L}_{\text {brane }}= & \delta_{0}\left[-\frac{a}{4} F^{\mu \nu} F_{\mu \nu}-\frac{c}{2} F^{\mu 5} F_{\mu 5}+\frac{c}{2} \partial^{\mu} \Sigma \partial_{\mu} \Sigma+\frac{a}{2} D^{2}-c \Sigma \partial_{y} D+c F_{\chi} F_{\chi}^{\dagger}\right. \\
& \left.-a \lambda_{1} i \sigma^{\mu} \partial_{\mu} \bar{\lambda}_{1}-c \lambda_{2} i \sigma^{\mu} \partial_{\mu} \bar{\lambda}_{2}+c \lambda_{2} \partial_{y} \lambda_{1}+c \bar{\lambda}_{2} \partial_{y} \bar{\lambda}_{1}\right] .
\end{aligned}
$$

Using the equations of motion for the auxiliary fields, $F_{\chi}=0$ and $(1+$ $\left.a \delta_{0}\right) D=-\partial_{y}\left[\left(1+c \delta_{0}\right) \Sigma\right]$, we find the following Lagrangian for the dynamical fields:

$$
\begin{aligned}
\mathcal{L}= & -\frac{1}{4}\left(1+a \delta_{0}\right) F^{\mu \nu} F_{\mu \nu}+\frac{1}{2}\left(1+c \delta_{0}\right) F^{\mu 5} F_{\mu 5} \\
& -\frac{1}{2}\left(1+c \delta_{0}\right) \partial^{\mu} \Sigma \partial_{\mu} \Sigma-\frac{1}{2} \frac{1}{1+a \delta_{0}} \partial_{y}\left[\left(1+c \delta_{0}\right) \Sigma\right] \partial_{y}\left[\left(1+c \delta_{0}\right) \Sigma\right] \\
& +\left(1+a \delta_{0}\right) \bar{\lambda}_{L} i \not \partial \lambda_{L}+\left(1+c \delta_{0}\right) \bar{\lambda}_{R} i \not \partial \lambda_{R}+\left(1+c \delta_{0}\right)\left(\bar{\lambda}_{R} \partial_{y} \lambda_{L}+\partial_{y} \bar{\lambda}_{L} \lambda_{R}\right),
\end{aligned}
$$

where we have defined the chiral 4-component spinors $\lambda_{L}^{T}=\left(\lambda_{1}, 0\right)$ and $\lambda_{R}^{T}=$ $\left(0, \bar{\lambda}_{2}\right)$. 
Again, the equations of motion for bosons and fermions in the same 4D supermultiplet are identical, which leads to the expected degeneracy of the spectra. Indeed, the relevant differential equations are

$$
\partial_{y}\left[\left(1+c \delta_{0}\right) \partial_{y}\right] f_{n}^{A}=-m_{n}^{2}\left(1+a \delta_{0}\right) f_{n}^{A},
$$

for the gauge boson,

$$
\partial_{y}^{2}\left[\left(1+c \delta_{0}\right) f_{n}^{S}\right]=\frac{a \delta_{0}^{\prime}}{1+a \delta_{0}} \partial_{y}\left[\left(1+c \delta_{0}\right) f_{n}^{S}\right]-m_{n}^{2}\left(1+a \delta_{0}\right) f_{n}^{S},
$$

for the scalar, and

$$
\begin{aligned}
& \partial_{y} f_{n}^{L}=-m_{n} f_{n}^{R}, \\
& \partial_{y}\left[\left(1+c \delta_{0}\right) f_{n}^{R}\right]=m_{n}\left(1+a \delta_{0}\right) f_{n}^{L},
\end{aligned}
$$

for the fermions. If we now iterate the first order fermionic differential equations we find that they are identical to the ones of their bosonic counterparts.

Using the results in the previous section we observe that the KK masses of all the fields are the same as the ones for gauge bosons. In particular, they do not exhibit a $b$-like behaviour ${ }^{5}$. This can be understood as the combination of gauge invariance, $\mathrm{N}=1$ supersymmetry relations and the fact that both chiralities combine to produce 4D Dirac masses. On the other hand, there is no finite solution when $c<0$, and the wave functions of even $\Sigma$ and $\lambda_{R}$ are forced to vanish at $y=0$ when $c>0$.

To summarize this section, supersymmetry improves the behaviour of the KK masses and wave functions, but it does not cure it.

\section{Singular solutions, the thin brane limit and perturbation theory}

We have seen that the brane operators which contain derivatives with respect to $y$ give rise to solutions behaving in a quite singular way. In this section we

\footnotetext{
${ }^{5}$ For even $\lambda_{R}$ this is so in the fermionic sector because supersymmetry fixes $a^{R}=c$.
} 
study in greater detail a simple example which exhibits the essential features of the general cases studied above.

Consider the following free Lagrangian of a 5D massless fermion:

$$
\begin{aligned}
\mathcal{L}= & \bar{\psi}_{L} i \not \partial \psi_{L}+\bar{\psi}_{R} i \not \partial \psi_{R}-\bar{\psi}_{L} \partial_{y} \psi_{R}+\bar{\psi}_{R} \partial_{y} \psi_{L} \\
& -\frac{b}{2} \delta_{0}\left[\bar{\psi}_{L} \partial_{y} \psi_{R}+\left(\partial_{y} \bar{\psi}_{R}\right) \psi_{L}\right] .
\end{aligned}
$$

We take $\psi_{L}$ even and $\psi_{R}$ odd. The coefficient of the brane term, $b$, is naturally of order $1 / \Lambda$. Once more, we assume that the delta function is regularized. The equations of motion can then be derived in the usual way. They read

$$
\begin{aligned}
& \left(1+\frac{b}{2} \delta_{0}\right) \partial_{y} \psi_{R}=i \not \partial \psi_{L} \\
& {\left[\left(1+\frac{b}{2} \delta_{0}\right) \partial_{y}+\frac{b}{2} \delta_{0}^{\prime}\right] \psi_{L}=-i \not \partial \psi_{R}}
\end{aligned}
$$

These equations admit a zero mode solution: $\psi_{L, R}(x, y)=f^{L, R}(y) \xi_{L, R}(x)$, with $\not \partial \xi_{L, R}=0$. This solution is chiral since the boundary conditions for the odd component, $\psi_{R}(0)=\psi_{R}(\pi R)=0$, together with (4.2) with a vanishing $r h s$, imply that $f^{R}=0$ everywhere. This leaves a homogeneous first order equation for $f^{L}$ with no further constraint. Using the following representation of the delta function,

$$
\delta(y)=\frac{\epsilon}{\pi\left(y^{2}+\epsilon^{2}\right)},
$$

we readily find

$$
\begin{aligned}
f^{L} & =N \frac{1}{1+\frac{b}{2} \delta_{0}} \\
& =2 N \pi \frac{y^{2}+\epsilon^{2}}{b \epsilon+2 \pi\left(y^{2}+\epsilon^{2}\right)}
\end{aligned}
$$

with $N$ a normalization constant. The first equation, (4.5), holds for any regularization of the delta function. Observe that when $b<0$ and $\epsilon \leq|b| / 2 \pi$ in (4.6), the function is singular at the points $y= \pm \sqrt{-\epsilon(b / 2+\pi \epsilon) / \pi}$; moreover, its integral is divergent. Therefore we have to restrict $b$ to nonnegative values. The limit $\epsilon \rightarrow 0$ then depends only on whether $b=0$ or $b>0$. When $b$ vanishes the limit of (4.5) is simply $f^{L}(y)=N$. For $b>0, f^{L}$ approaches 
$N$ everywhere except around $y=0$, where it goes to zero in such a way that $\int \mathrm{d} y \delta(y) f^{L}(y) \rightarrow 0$. Therefore, for positive $b$ the zero mode of the fermion does not couple to fields living on the brane. Moreover, it is insensitive to other possible brane terms and to the precise (positive) value of $b$ itself.

Obviously, in the thin brane limit, the zero mode is not smooth in the coefficient $b$ : the solution for a small positive value of $b$ differs drastically (around the brane) from the one for vanishing $b$, and there is no finite solution for any negative $b$. On the other hand, for a thick brane with $\epsilon>|b| / 2 \pi$, the zero mode is well defined and smooth in $b$.

This behaviour also shows up in the limit of the perturbative expansion in $1 / \Lambda$ of the finite $\epsilon$ result (4.5):

$$
f^{L}=N\left[1+\sum_{n=1}^{\infty}\left(-\frac{1}{2}\right)^{n} \delta_{0}^{n} b^{n}\right] .
$$

We see that the breaking of perturbation theory is caused by the functions $\delta_{0}^{n}, n>1$, which are singular when $\epsilon \rightarrow 0$. Strictly, the expansion (4.7) is only valid for small $|b| / \epsilon$, and therefore it is incompatible with the limit $\epsilon \rightarrow 0$. However, the situation is analogous to the one in standard (renormalizable or nonrenormalizable) quantum field theories, where the quantum UV divergences make the loop expansion badly defined. Nevertheless, in these theories it is well known that one can work order by order after renormalization: each order is finite and (at weak coupling) smaller than the lower ones. Since our classical divergences have essentially the same origin as the usual quantum ones (the unknown UV of the theory), it is natural to apply the same renormalization procedure to obtain well defined, finite predictions at each order in perturbation theory. We develop this point of view in the next section.

\section{Renormalization and critical theories}

The divergences we have encountered in perturbation theory are actually a common feature of field theories with infinitely thin defects. They appear, 
for example, in classical electrodynamics with point sources. These singularities indicate a breakdown of the effective theory at scales in which the details of the fundamental theory - for instance, in our case, a finite thickness of the brane or stringy effects - cannot be neglected. In this sense, they are analogous to the usual UV divergences in radiative corrections. In [22], Goldberger and Wise have shown how to deal with classical divergences in brane theories of codimension greater than one by the usual renormalization procedure of quantum field theory. The same idea can be applied to brane theories of codimension one with more singular operators: adding appropriate counterterms it is possible to cancel the $\delta(0)$ singularities order by order. Let us see how this can be achieved to $\mathcal{O}\left(1 / \Lambda^{2}\right)$ in the example of the previous section. In this simple case it is sufficient to add to the Lagrangian the following counterterms:

$$
\mathcal{L}_{\text {ct }} \supset-\frac{b^{2}}{4} \delta_{0}^{2} \bar{\psi}_{L} \mathrm{i} \not \partial \psi_{L}-\frac{b^{2}}{4} \delta_{0}^{2}\left(\bar{\psi}_{L} \partial_{y} \psi_{R}+\text { h.c. }\right) .
$$

Then, it is straigthforward to check that, to second order, the zero mode solution is well defined:

$$
f^{L}=N\left(1-\frac{b}{2} \delta_{0}+\mathcal{O}\left(1 / \Lambda^{3}\right)\right),
$$

and the kinetic term in the action is finite:

$$
\int_{-\pi R}^{\pi R} \mathrm{~d} y\left(1-\frac{b^{2}}{4} \delta_{0}^{2}\right)\left(f^{L}\right)^{2}=N^{2}\left(2 \pi R-b+\mathcal{O}\left(1 / \Lambda^{3}\right)\right) .
$$

This example is quite trivial, but it illustrates the main properties of the counterterms:

1. They are operators of higher dimension. This is related to the fact that the brane coefficients are dimensionful and the theory is nonrenormalizable.

2. The divergences are not pure numbers, but functions of $y$ localized at the branes, i.e., functions like $\delta_{0}^{2}$. Notice that also one loop corrections in an orbifold without brane terms require the introduction of counterterms in a nontrivial background: the delta functions at the fixed points. 
3. The coefficients of these singular operators are determined (in a given regularization) by the requirement that the divergences must be cancelled. This is analogous to the fact that the infinite parts of usual counterterms are fixed by the structure of the radiative corrections. However, in our case there is no freedom to adjust at will the finite part of the coefficients, as the operator itself is "infinite". This has a very important consequence:

4. The coefficients of the higher dimensional operators with more than one delta are not independent parameters of the effective theory. They do not require new observables to be fixed by experiment.

Of course, a full calculation at a given order will include not only the classical part we have considered so far, but also loop diagrams and, possibly, contributions from higher order operators in the effective action. All these contributions may include new thin brane singularities, which should be cancelled. This can be achieved adjusting the coefficients of the counterterms accordingly. In particular, they will contain quantum infinities. In this way, the usual quantum divergences and the thin brane singularities are subtracted all at once.

It should be noted that the renormalization procedure we propose is not identical to the one in [22, where the classical divergences are just numbers which can be absorbed into the bare first order couplings. In fact, the 5D theories we are analising differ from the 6D model in [22] in at least two important aspects. First, we find power-law, instead of logarithmic divergences. Second, we are dealing with operators with a richer structure, due to the inclusion of derivatives orthogonal to the branes. This seems to require in the counterterms nontrivial backgrounds with more than one delta function.

We would also like to comment on the similarities and differences of our approach to the one in [16]. There, the singularities in 5D theories with brane operators, like the ones we are discussing, were analised in perturbation theory. The authors proposed to substitute the singular brane terms by terms without $y$ derivatives, giving the same solutions to the equations of motion outside the core of the brane. The resulting action was interpreted as 
a renormalized action. However, we observe that this "massage" (we follow the terminology of [16]) by itself does not lead to finite solutions since in the original action the divergences near the branes induce divergences also for the solutions far from the branes, and even for observables such as KK masses. In the "massaged" theory this divergent behaviour comes from explicit divergences in the coefficients of the nonsingular operators, which translate into divergencies in the boundary conditions just outside the core of the branes. On the other hand, subtracting the explicit divergences in the mentioned coefficients leads to really finite results. This can be achieved by adding counterterms such as the ones in (5.1), which has the additional advantage that the behaviour inside the brane is also well defined. We should remark that, in their application of "massaging" to the study of the stability of the compact Randall-Sundrum model, the authors of [16] considered arbitrary finite coefficients of operators without $y$ derivatives. Hence, they did use a renormalized theory.

In Appendix B we work out the renormalization of a more involved example: the propagator of a scalar field in the presence of a " $b$ " brane term. A more complete study of renormalization of theories with brane terms will be presented elsewhere.

This renormalization process can be interpreted in a complementary way. We have seen that, in general, theories with brane kinetic terms have a singular behaviour in the thin brane limit (before renormalization), such that perturbation theory is spoiled. However, there is a subclass of "critical" theories which are well behaved and predictive: the ones with a tower of operators identical to the counterterms introduced above (plus all the operators present initially). The coefficients of these operators are not independent parameters: both their finite part and the part depending on the quantum renormalization scale are functions of the coefficients of bulk operators and brane operators with only one delta. In this sense, these critical effective theories correspond to a particular reduction of couplings [23, 24]. In fact, such relations should exist in the low energy effective theory if the fundamental theory is to be well behaved for infinitely thin branes. For instance, in 17] it was hoped that M-theory would directly give well defined quantities 
without the $\delta(0)$ that were observed in the supergravity description of the Hořava-Witten model. ${ }^{6}$ A particularly simple example of critical theory (at least at the classical level), in which all the coefficients of operators of order higher than one vanish, is the one for a gauge boson with $c=0$. Indeed, in this case one can directly perform the KK reduction to all orders in $a$, as done in [5] and in Section 2.

\section{Conclusions}

We have studied 5D theories for spins $\leq 1$ with general brane kinetic terms. We have shown that some terms which had been neglected in the past play an active role, especially in the case of fermions. We have first calculated the KK masses and wave functions in these theories to all orders in the coefficients of the brane terms, using a combination of analytical and numerical methods. We have found that some terms change smoothly the solutions without brane terms, while others - the ones with derivatives with respect to $y$-change them abruptly, destroying the perturbative hierarchy of the effective theory. We have also built supersymmetric free actions with brane terms for the hyper and vector multiplets and studied their KK decompositions. Supersymmetry does not solve in general the difficulties produced by the second kind of operators, although it can alleviate them in some cases. We have then discussed in detail a particular case, showing that the singular behaviour is caused by the presence of infinitely thin branes. If we insist on performing perturbative calculations, we have to face $\delta(0)$-like divergences at $\mathcal{O}\left(1 / \Lambda^{2}\right)$ and higher orders. At this point, at least three different paths can be followed.

First, we can simply take the exact results at their faith value and ac-

\footnotetext{
${ }^{6}$ It might be objected that supersymmetric theories cannot be critical — or, equivalently, renormalization breaks supersymmetry - since in our discussion above supersymmetry determined completely the higher order operators of the on-shell theory, and we have seen that they do not eradicate the singular behaviour. This is actually not so, since we included only first order operators in the off-shell action. Higher order off-shell operators compatible with supersymmetry can give rise to a critical theory. Of course, the on-shell supersymmetry transformations will be different from the ones without the new operators.
} 
cept a theory with such behaviour. From a phenomenological point of view, the possible realistic models would be constrained by the decoupling of the wave functions from the branes and, in the nonsupersymmetric scenario, the absence of scalar zero modes. In particular, bulk fermions would not couple to a boundary Higgs, while a nonsupersymmetric bulk Higgs would not have the required zero mode. Even more problematic is the fact that perturbation theory breaks down. It seems very difficult, if not impossible, to construct a predictive interacting field theory in extra dimensions without some hierarchy controlling the size of the operators.

Second, we can work with thick branes instead of zero width branes (see 25] for recent calculations with kinetic terms on thick branes). This is natural if the brane is a domain wall. The disadvantage of this approach is that the results depend on the substructure of the brane and are thus very

model dependent. Furthermore, we would like to be able to define sensible field theories in orbifold compatifications with vanishing brane width.

Third, we can renormalize the theory to render it free from both classical and quantum divergences in perturbation theory. This is equivalent to working with a "critical" theory containing a tower of operators whose coefficients satisfy certain relations. Here we have just argued that this is a sensible approach to construct extra dimensional models with thin branes. We have not described the renormalization program in detail nor proven that it can be carried out to all orders. This subject is under investigation.

Including mass terms in the bulk and on the branes may be relevant for phenomenology, but it does not alter in an essential way the results given here. The same holds for brane kinetic branes on the second brane. Finally, in a complete model one should also take into account brane interactions, which may require the introduction of new counterterms.

\section{Acknowledgements}

We thank Ferruccio Feruglio, Luca Girlanda, Ignacio Navarro, Toni Riotto and Verónica Sanz for discussions. This work has been supported in part by MCYT under contract FPA2000-1558, by Junta de Andalucía group FQM 
101, by the European Community's Human Potential Programme under contract HPRN-CT-2000-00149 Physics at Colliders, and by PPARC.

\section{A First order solutions}

In this appendix we present the KK expansions to first order in $1 / \Lambda$. The first order results are directly finite, but they are only meaningful when the theory is renormalized, so that higher orders are also finite. Perturbatively, the solutions to the generalized eigenvalue problem can be computed in different ways. If the full analytic solutions are known for a smooth regularization of the brane, we can expand them in powers of the dimensionful coefficients $a, b, c$ and then take the limit $\epsilon \rightarrow 0$. Alternatively, we can perform the perturbative expansion from the very beginning in the eigenvalue equations and normalization conditions and solve them order by order (again for a finite $\epsilon$ and taking the limit of thin brane only at the end of the calculation).

We find that the first order KK masses are invariant under $a \leftrightarrow-b$ for scalars and under $a^{L} \leftrightarrow-b$ and $a^{R} \leftrightarrow-c$ for fermions, while the wave functions are invariant up to possible delta functions. This can be understood in the following way. In the scalar sector, one can perform the field redefinition

$$
\phi \rightarrow\left(1-\frac{b}{2} \delta_{0}\right) \phi,
$$

which to first order only has the effect of redefining the coefficients $b \rightarrow 0$ and $a \rightarrow a-b$. In the fermion sector, the field redefinitions

$$
\begin{aligned}
& \psi_{L} \rightarrow\left(1-\frac{b}{2} \delta_{0}\right) \psi_{L}, \\
& \psi_{R} \rightarrow\left(1-\frac{c}{2} \delta_{0}\right) \psi_{R},
\end{aligned}
$$

redefine $b \rightarrow 0, a^{L} \rightarrow a^{L}-b$ and $c \rightarrow 0, a^{R} \rightarrow a^{R}-c$. All these field redefinitions generate higher order operators as well. These are responsible for the singularities observed at higher orders. 


\section{Scalars and Gauge Bosons}

Consider an even scalar with general values of $a, b, c$ (gauge bosons satisfy the same equations with the condition $b=0$ ). The solution to first order is, for the zero mode,

$$
f_{0}(y)=1+\frac{b-a}{4 \pi R}-\frac{b}{2} \delta_{0},
$$

and for the massive modes,

$$
\begin{aligned}
f_{n}(y)= & \sqrt{2} \cos \left(m_{n}^{(0)} y\right)-\frac{b}{\sqrt{2}} \delta_{0} \\
& +\frac{1}{\sqrt{2}} \frac{b-a}{2 \pi R}\left[\cos \left(m_{n}^{(0)} y\right)+2 m_{n}^{(0)}(\pi R-|y|) \sin \left(m_{n}^{(0)}|y|\right)\right],
\end{aligned}
$$

with $m_{n}^{(0)}=n / R$, and masses

$$
m_{n}=\frac{n}{R}\left(1+\frac{b-a}{2 \pi R}\right) .
$$

Note that the "odd-odd" parameter $c$ has no effect to this order.

For an odd scalar there is no zero mode and the massive ones are

$$
\begin{aligned}
f_{n}(y)= & \sqrt{2} \sin \left(m_{n}^{(0)} y\right) \\
& +\frac{1}{\sqrt{2}} \frac{c}{2 \pi R}\left[\sin \left(m_{n}^{(0)} y\right)-2 m_{n}^{(0)} \sigma(y)(\pi R-|y|) \cos \left(m_{n}^{(0)} y\right)\right]
\end{aligned}
$$

with $\sigma(y)$ the sign function and masses

$$
m_{n}=\frac{n}{R}\left(1+\frac{c}{2 \pi R}\right) .
$$

The "odd-odd" parameters, which are now $a$ and $b$ do not contribute to this order while the "even-even" parameter $c$ has a non-trivial effect, in contrast with the results in Section 2 .

\section{Fermions}

For the even component (which we take here to be the LH component), this case is identical to the scalar case except for the term with $a^{R}$, which, just 
like $c$, does not contribute to first order. The wave functions and masses to $\mathcal{O}(1 / \Lambda)$ are

$$
f_{0}^{L}(y)=1+\frac{b-a^{L}}{4 \pi R}-\frac{b}{2} \delta_{0},
$$

for the zero mode (with no zero mode for the odd chirality), and

$$
\begin{aligned}
f_{n}^{L}(y)= & \sqrt{2} \cos \left(m_{n}^{(0)} y\right)-\frac{b}{\sqrt{2}} \delta_{0} \\
& +\frac{1}{\sqrt{2}} \frac{b-a^{L}}{2 \pi R}\left[\cos \left(m_{n}^{(0)} y\right)+2 m_{n}^{(0)}(\pi R-|y|) \sin \left(m_{n}^{(0)}|y|\right)\right] \\
f_{n}^{R}(y)= & \sqrt{2} \sin \left(m_{n}^{(0)} y\right) \\
& +\frac{1}{\sqrt{2}} \frac{b-a^{L}}{2 \pi R}\left[\sin \left(m_{n}^{(0)} y\right)-2 m_{n}^{(0)} \sigma(y)(\pi R-|y|) \cos \left(m_{n}^{(0)} y\right)\right] \\
m_{n}= & \frac{n}{R}\left(1+\frac{b-a^{L}}{2 \pi R}\right)
\end{aligned}
$$

for the massive modes.

\section{B Renormalization of the scalar propagator}

Here we give another example of classical renormalization. We consider the scalar theory (2.1) with $a=c=0$ for an even scalar, and calculate perturbatively the classical propagator to $\mathcal{O}\left(1 / \Lambda^{2}\right)$, i.e., $\mathcal{O}\left(b^{2}\right)$. We work in momentum space for the first four coordinates and in position space for the 5 th coordinate. The brane delta functions are, as usual, assumed to be regularized and this time we indicate this explicitly through a subindex $\epsilon$, to differentiate these deltas from the ones which do not need regularization. In perturbation theory, the propagator can be written

$$
\Delta\left(p ; y_{1}, y_{2}\right)=\Delta_{0}\left(p ; y_{1}, y_{2}\right)+\Delta_{1}\left(p ; y_{1}, y_{2}\right)+\Delta_{2}\left(p ; y_{1}, y_{2}\right)+\mathcal{O}\left(b^{3}\right)
$$

with $\Delta_{n}$ proportional to $b^{n}$. The zeroth order propagator satisfies the equation

$$
\left[\partial_{y_{1}}^{2}+p^{2}\right] \Delta_{0}\left(p ; y_{1}, y_{2}\right)=-\frac{1}{2}\left[\delta\left(y_{1}-y_{2}\right)+\delta\left(y_{1}+y_{2}\right)\right],
$$


with boundary conditions

$$
\begin{aligned}
& {\left[\partial_{y_{1}} \Delta_{0}\left(p ; y_{1}, y_{2}\right)\right]_{y_{1}=0^{+}}=0, y_{2} \neq 0} \\
& {\left[\partial_{y_{1}} \Delta_{0}\left(p ; y_{1}, y_{2}\right)\right]_{y_{1}=\pi R^{-}}=0, y_{2} \neq \pi R}
\end{aligned}
$$

The solution is

$$
\Delta_{0}\left(p ; y_{1}, y_{2}\right)=-\frac{\cos \left(p y_{<}\right) \cos \left(p\left(\pi R-y_{>}\right)\right)}{2 p \sin (p \pi R)},
$$

with $y_{<}=\operatorname{Min}\left\{\left|y_{1}\right|,\left|y_{2}\right|\right\}, y_{>}=\operatorname{Max}\left\{\left|y_{1}\right|,\left|y_{2}\right|\right\}$. The first order propagator is given by the zeroth order propagator with one insertion of the brane kinetic term. It reads

$$
\begin{aligned}
\Delta_{1}\left(p ; y_{1}, y_{2}\right)= & \frac{b}{2} \int \mathrm{d} z \delta_{\epsilon}(z) \Delta_{0}\left(p ; y_{1}, z\right)\left(\overleftarrow{\partial_{z}^{2}}+\partial_{z}^{2}\right) \Delta_{0}\left(p ; z, y_{2}\right) \\
\rightarrow & \frac{b}{2}\left[-2 p^{2} \Delta_{0}\left(p ; y_{1}, 0\right) \Delta_{0}\left(p ; 0, y_{2}\right)-\delta\left(y_{1}\right) \Delta_{0}\left(p ; y_{1}, y_{2}\right)\right. \\
& \left.\quad-\Delta_{0}\left(p ; y_{1}, y_{2}\right) \delta\left(y_{2}\right)\right]
\end{aligned}
$$

In the second line we have used $(\underline{B .2})$ and taken the limit $\epsilon \rightarrow 0$, which is well defined. The singularities arise at the next order. The second order propagator has two insertions of the brane kinetic term:

$$
\begin{aligned}
& \Delta_{2}\left(p ; y_{1}, y_{2}\right)=\frac{b^{2}}{4} \int \mathrm{d} z_{1} \mathrm{~d} z_{2} \delta_{\epsilon}\left(z_{1}\right) \delta_{\epsilon}\left(z_{2}\right) \Delta_{0}\left(p ; y_{1}, z_{1}\right)\left(\overleftarrow{\partial_{z_{1}}^{2}}+\partial_{z_{1}}^{2}\right) \Delta_{0}\left(p ; z_{1}, z_{2}\right) \\
& \times\left(\overleftarrow{\partial_{z_{2}}^{2}}+\partial_{z_{2}}^{2}\right) \Delta_{0}\left(p ; z_{2}, y_{2}\right)
\end{aligned}
$$

Using (B.2) for the internal propagator and integrating on $z_{2}$, we find a contribution

$$
\begin{aligned}
{\left[\Delta_{2}\left(p ; y_{1}, y_{2}\right)\right]_{\mathrm{div}}=\frac{b^{2}}{4} \int } & \mathrm{d} z_{1}\left\{p^{2} \delta_{\epsilon}^{2}\left(z_{1}\right) \Delta_{0}\left(p ; y_{1}, z_{1}\right) \Delta_{0}\left(p ; z_{1}, y_{2}\right)\right. \\
& +\partial_{z_{1}}\left[\delta_{\epsilon}\left(z_{1}\right) \Delta_{0}\left(p ; y_{1}, z_{1}\right)\right] \partial_{z_{1}}\left[\delta_{\epsilon}\left(z_{1}\right) \Delta_{0}\left(p ; z_{1}, y_{2}\right)\right] \\
& \left.-\delta_{\epsilon}^{2}\left(z_{1}\right)\left[\Delta_{0}\left(p ; y_{1}, z_{1}\right)\left(\overleftarrow{\partial_{z_{1}}^{2}}+\partial_{z_{1}}^{2}\right) \Delta_{0}\left(p ; z_{1}, y_{2}\right)\right]\right\}
\end{aligned}
$$


which diverges when $\epsilon \rightarrow 0$. To renormalize the theory to second order (at the classical level) we add the following counterterms to the Lagrangian:

$$
\mathcal{L}_{\mathrm{ct}}=\frac{b^{2}}{4}\left\{-\delta_{\epsilon}^{2}(y) \partial_{\mu} \phi^{\dagger} \partial^{\mu} \phi-\partial_{y}\left[\delta_{\epsilon}(y) \phi^{\dagger}\right] \partial_{y}\left[\delta_{\epsilon}(y) \phi\right]+\delta_{\epsilon}^{2}(y)\left(\phi^{\dagger} \partial_{y}^{2} \phi+\partial_{y}^{2} \phi^{\dagger} \phi\right)\right\} .
$$

Then, the divergences are cancelled and taking $\epsilon \rightarrow 0$ we find the finite result

$$
\Delta_{2}^{\mathrm{ren}}\left(p ; y_{1}, y_{2}\right)=\frac{b^{2}}{4}\left[2 p^{2} \Delta_{0}\left(p ; y_{1}, 0\right)+\delta\left(y_{1}\right)\right] \Delta_{0}(p ; 0,0)\left[2 p^{2} \Delta_{0}\left(p ; 0, y_{2}\right)+\delta\left(y_{2}\right)\right]
$$

We have checked that the counterterms (B.9) also render the KK reduction finite to order $\mathcal{O}\left(1 / \Lambda^{2}\right)$. The explicit delta functions in the propagator can be translated through a field redefinition into brane interaction terms. For points $y_{1}$ and $y_{2}$ away from the first brane, the renormalized classical propagator reads

$$
\begin{aligned}
\Delta^{\mathrm{ren}}\left(p ; y_{1}, y_{2}\right)= & \Delta_{0}\left(p ; y_{1}, y_{2}\right)-b p^{2} \Delta_{0}\left(p ; y_{1}, 0\right) \Delta_{0}\left(p ; 0, y_{2}\right) \\
& +b^{2} p^{4} \Delta_{0}\left(p ; y_{1}, 0\right) \Delta_{0}(p ; 0,0) \Delta_{0}\left(p ; 0, y_{2}\right)+\mathcal{O}\left(b^{3}\right) \\
= & -\frac{\left[\cos \left(p y_{<}\right)+\frac{b}{2} p \sin \left(p y_{<}\right)\right] \cos \left(p\left(\pi R-y_{>}\right)\right)}{2 p\left[\sin (p \pi R)-\frac{b}{2} p \cos (p \pi R)\right]}+\mathcal{O}\left(b^{3}\right) .
\end{aligned}
$$

Actually, working out the renormalization to all orders and resumming the series, it can be shown that the exact renormalized propagator is equal to the explicit expression in the last line of (B.11) 26]. The KK masses and wave functions can be easily obtained from (B.11): they are the poles and the square roots of the residua of the propagator. They agree with the ones for an even scalar when $b=c=0$ and $a=-b$. In fact, the explicit expression in (B.11) is identical to the exact propagator of the theory with $b=c=0$ and $a=-b$. As we have observed in Appendix A, the field redefinition (A.1) eliminates the $b$ operator from the action (2.1) and redefines $a \rightarrow a-b$. It also introduces new operators at higher orders, which give rise to singularities. The result in (B.11) shows that performing the field redefinition (A.1) in the action with the counterterms (B.9), the second order terms cancel out (at 
least when $a=c=0$ initially). Conversely, performing the field redefinition and neglecting the higher order operators which are generated amounts to a renormalization of the theory. The delta functions in (A.1) account for the ones in $\Delta^{\text {ren }}$.

\section{References}

[1] P. Horava and E. Witten, Nucl. Phys. B 460, 506 (1996) arXiv:hep-th/9510209.

[2] E. A. Mirabelli and M. E. Peskin, Phys. Rev. D 58, 065002 (1998) arXiv:hep-th/9712214.

[3] G. R. Dvali, G. Gabadadze and M. Porrati, Phys. Lett. B 485, 208 (2000) arXiv:hep-th/0005016.

[4] H. Georgi, A. K. Grant and G. Hailu, Phys. Lett. B 506, 207 (2001) arXiv:hep-ph/0012379.

[5] M. Carena, T. M. Tait and C. E. Wagner, Acta Phys. Polon. B 33, 2355 (2002) arXiv:hep-ph/0207056.

[6] G. R. Dvali, G. Gabadadze and M. A. Shifman, Phys. Lett. B 497, 271 (2001) arXiv:hep-th/0010071.

[7] H. C. Cheng, K. T. Matchev and M. Schmaltz, Phys. Rev. D 66, 036005 (2002) arXiv:hep-ph/0204342.

[8] G. von Gersdorff, N. Irges and M. Quiros, Nucl. Phys. B 635, 127 (2002) arXiv:hep-th/0204223.

[9] M. Carena, E. Ponton, T. M. Tait and C. E. Wagner, arXiv:hep-ph/0212307.

[10] H. Davoudiasl, J. L. Hewett and T. G. Rizzo, arXiv:hep-ph/0212279.

[11] F. del Aguila and J. Santiago, arXiv:hep-ph/0212205. 
[12] A. Hebecker, Nucl. Phys. B 632, 101 (2002) arXiv:hep-ph/0112230.

[13] H. C. Cheng, K. T. Matchev and M. Schmaltz, Phys. Rev. D 66, 056006 (2002) arXiv:hep-ph/0205314.

[14] B. s. Kyae, arXiv:hep-th/0207272.

[15] M. Chaichian and A. Kobakhidze, arXiv:hep-ph/0208129

[16] A. Lewandowski and R. Sundrum, Phys. Rev. D 65, 044003 (2002) arXiv:hep-th/0108025.

[17] P. Horava and E. Witten, Nucl. Phys. B 475, 94 (1996) arXiv:hep-th/9603142.

[18] K. A. Meissner, H. P. Nilles and M. Olechowski, Acta Phys. Polon. B 33, 2435 (2002) arXiv:hep-th/0205166.

[19] J. A. Bagger, F. Feruglio and F. Zwirner, Phys. Rev. Lett. 88, 101601 (2002) arXiv:hep-th/0107128.

[20] N. Arkani-Hamed, T. Gregoire and J. Wacker, JHEP 0203, 055 (2002) arXiv:hep-th/0101233.

[21] D. Marti and A. Pomarol, Phys. Rev. D 64, 105025 (2001) arXiv:hep-th/0106256.

[22] W. D. Goldberger and M. B. Wise, Phys. Rev. D 65, 025011 (2002) arXiv:hep-th/0104170.

[23] W. Zimmermann, Commun. Math. Phys. 97, 211 (1985).

[24] R. Oehme and W. Zimmermann, Commun. Math. Phys. 97, 569 (1985).

[25] M. Kolanovic, arXiv:hep-th/0301116.

[26] F. del Aguila, M. Pérez-Victoria and J. Santiago, in preparation. 